\title{
Dominancia Cerebral en la Industria Maquiladora en Ciudad Juárez, Chihuahua, México
}

\author{
Brain Dominance in the Maquiladora Industry of Juarez city, México
}

\author{
Alberto Castro ${ }^{1 *}$, Ivonne Gaucín ${ }^{1}$, María González ${ }^{1}$, María Gaytán $^{1}$, Patricia Vázquez ${ }^{1}$ \& Arturo Ávila ${ }^{2}$ \\ 1 Departamento de Ciencias Sociales, Universidad Autónoma de Ciudad Juárez. Ciudad de Juárez, México \\ 2 Departamento de Recursos Humanos, Empresa Maquiladora de exportación. Ciudad de Juárez, México
}

\section{Resumen}

El objetivo del estudio fue identificar modelos de pensamiento y habilidades en correspondencia al puesto y área laboral, mediante la evaluación del instrumento Herrmann de Dominancia Cerebral HBDI (Herrmann Brain Dominance Instrument, 1989), en una planta industrial de Ciudad Juárez, Chih. Participaron 176 gerentes y personal administrativo mexicanos. Se describen estadísticos de cuatro categorías de dominancia en tres áreas laborales. Se encontraron puntuaciones elevadas en las categorías lógicocontrolador para todas las áreas laborales respecto a las habilidades interpersonales-holísticas, lo cual corresponde al manejo de dominancia cerebral del hemisferio cortical superior. Con una varianza total explicada del $76 \%$ en factorial confirmatorio y fiabilidad aceptable de la escala $(\alpha=.657)$, el instrumento fue identificado como versátil, económico y de fácil evaluación, al servir adecuadamente para selección, promoción, desarrollo organizacional y comprensión del clima laboral.

Palabras clave: dominancia cerebral, industria maquiladora

\begin{abstract}
The objective of the study was to identify models of thought and abilities in correspondence to the position and labor area by means of the evaluation of the instrument Herrmann de Cerebral Dominance HBDI (Herrmann Brain Dominance Instrument, 1989), in an industrial plant of City Juarez, Chih. To 176 Mexican managers and administrative personnel participated. They describe themselves statistical of four categories of dominance in three labor areas. Were scores elevated in the categories logical-controller for all the labor areas with respect to the interpersonal-holistic abilities, which corresponds to the handling of cerebral dominance of the superior cortical hemisphere. With an explained total variance of confirming factorial $76 \%$ in and acceptable reliability of the scale $(\alpha=.657)$, the instrument was identified like versatile, economic and of easy evaluation when being used suitably for selection, promotion, organizational development and understanding of the labor climate.
\end{abstract}

Keywords: brain dominance, maquiladora industry

\footnotetext{
* Correspondencia: alcastro@uacj.mx Universidad Autónoma de Ciudad Juárez, Av. Universidad y H.C. Militar zona Chamizal C.P. 32000, Ciudad de Juárez,Chihuahua, México

Recibido: 17-05-2011. Revisión desde: 18-05-11. Aceptado: 30-06-2011

DOI: $10.5839 / \mathrm{rcnp} .2011 .0601 .09$
} 


\section{Introducción}

La industria maquiladora requiere de nuevos procesos de evaluación de los recursos humanos, no solo para mejorar la productividad industrial, sino para garantizar la permanencia y estabilidad económica familiar de los trabajadores de niveles gerenciales y administrativos. En psicología laboral, el uso de instrumentos psicométricos en los procesos de selección, promoción y desarrollo organizacional, han sido considerados necesarios al reconocer la influencia de factores psicosociales como el liderazgo, clima laboral, productividad y competencias, entre otros. Sin embargo, los desarrollos científicos de las neurociencias, la programación neurolingüística y la lateralidad o dominancia de los hemisferios cerebrales han representado nuevos retos inter-transdisciplinarios para identificar habilidades productivas de empleados en las grandes industrias. Ciudad Juárez ha sido un espacio socioestratégico para el desarrollo industrial desde mediados del siglo $X X$, donde equidistan nodos económicos y productivos de grandes consorcios de compañías extranjeras. Actualmente, más de 350 industrias maquiladoras se encuentran activas, considerando una disminución de actividades económicas generales en el último trienio a causa de la violencia social y atentados del crimen organizado, lo cual ha revertido la tasa de crecimiento poblacional después que fenómenos como la migración la habían fortalecido.

En las industrias juarenses, más de la mitad de la fuerza de trabajo está compuesta por gente que produce, procesa y transforma información. Las teorías del managment, como parte de las neurociencias, han evolucionado a pasos agigantados, pero la fusión de ellas, en la práctica, aún no se ha concentrado totalmente para afrontar la nueva realidad laboral (Bunderson \& Olsen, 1980). Así, el concepto original de dominancia cerebral se basó en la idea de que un hemisferio (izquierdo o derecho) dominaría el control del comportamiento. La dominancia cerebral se especificó como la tendencia de un hemisferio a procesar la información y tomar el control al dar una respuesta determinada (Springer \& Deutsch, 2006). El hemisferio izquierdo se ha reconocido como el procesador de secuencias racionales específicas, en consecuencia, determina procesos analíticos sistemáticos, lógicos y convergentes. Por contraste, algunas áreas del hemisferio derecho se adaptan más al procesamiento simultáneo para percibir patrones espaciales y sus relaciones, lo cual determina procesos analíticos de tipo holísticos o sistémicos generales, creativos y divergentes (Bunderson, Olsen \& Herrmann, 1982).

El desarrollo de la ciencia ha dado pauta para planear, organizar y controlar el capital humano de una forma cada vez más eficiente. Los factores de producción, rentabilidad y crecimiento, mediante el aprovechamiento del potencial del personal, han sido relevantes ante la propia gestión del conocimiento (Robbins, 1999). De acuerdo con Roger Sperry (1973), el hemisferio izquierdo es responsable de la coordinación verbal, del pensamiento lineal, analítico y racional, y al hemisferio derecho le corresponden habilidades holísticas, conceptuales y espaciales. Asimismo, Ned Herrmann (1989) definió un modelo cerebral de cuatro factores para explicar el proceso de pensamiento y creación, como fusión de los hemisferios izquierdo-derechos planteados previamente en el modelo Triune Brain por MacLean (1990). La arquitectura del modelo Herrmann planteó la división del cerebro en dos cuadrantes corticales superiores y dos límbicos inferiores (Herrmann, 1996), cada uno asociado con un estilo particular de pensar, crear y aprender, así como con determinadas habilidades laborales, todo ello considerando la teoría de las inteligencias múltiples (Gardner, 1993), la cual expone la dominancia de determinadas habilidades verbales, numéricas, espaciales, musicales, interpersonales, intrapersonales y emocionales, entre otras.

\section{Método}

\section{Participantes}

En una industria maquiladora de exportación, dedicada a la producción de fibra óptica para el abastecimiento mundial de tecnologías de comunicación, participaron 176 gerentes y administrativos mexicanos de tres categorías laborales: Administrativos $A D(n=29,16,5 \%)$, Manejo de Personal MP ( $n=$ $60,34 \%$ ) y Diseño DS ( $n=87,49,5 \%)$. Estas categorías se agruparon a partir de actividades contractuales para cada puesto de trabajo de diez departamentos (Ramírez, 1986; Morrisey, 1996; Montoya, 2002). Con una edad promedio de 42 años, el $73,9 \% \quad(n=130)$ fueron hombres y $26,1 \% \quad(n=46)$ mujeres. En el área Administrativa (AD) se agruparon actividades de: manejo de documentos, planeación, distribución, abastecimiento y embarque de materiales o productos, así como el manejo de activos, control presupuestal, coordinación de áreas de compra y coordinación de la logística e identificación de problemas. En el área de Manejo de Personal (MP) se agruparon los departamentos de: recursos humanos, compras, producción y seguros; con actividades como el análisis de puestos, reclutamiento, selección, administración de sueldos y evaluación del desempeño; también, la revisión y actualización de bases de datos y negociación de descuentos, control de accidentes en el trabajo, enfermedades y procesos ergonómicos mediante el adiestramiento de métodos de trabajo. Por último, el área de Diseño (DS) fue categoría de agrupación de los departamentos de: ingeniería, sistemas y operaciones, donde se realizan actividades que determinan el proceso de fabricación, secuencia y calidad de operaciones, elaboración de planos de distribución y diseños de dispositivos materiales (ver tabla 1 ).

\section{Instrumentos}

Se utilizó el instrumento Herrmann de Dominancia Cerebral HBDI (Herrmann Brain Dominance Instrument, 1989) para identificar estilos de pensamiento $y$ habilidades percibidas según la dominancia cerebral de los participantes en los puestos de trabajo gerencial y administrativo, delimitados por la organización industrial. Con 120 adjetivos autoevaluativos se describen tipos de conducta organizadas en diez columnas con una ponderación del 4 al 1 en orden jerárquico: 4 corresponde a la descripción jerárquicamente más dominante, hasta el número 1 , el calificativo más débil. Se obtiene un perfil de dominancia cerebral identificado en cuatro factores: dos corticales superiores y dos límbicos inferiores - asociados a determinadas habilidades y formas de pensamiento laboraldefinidos como: matemáticos, analíticos, técnicos, lógicos y que resuelven problemas (A Lógico); confiables, planificadores, conservadores, administrativos (B Controlador); cuidadosos, 
Castro, et al. Rev. Chil. Neuropsicol. 6(1): 55-59, 2011

expresivos, interpersonales y sociables (C Interpersonal), y Holístico). artísticos, intuitivos, holísticos, futuristas e imaginativos (D

\begin{tabular}{|c|c|c|c|c|c|c|}
\hline \multirow[b]{2}{*}{ Área Laboral } & \multirow[b]{2}{*}{ Departamento } & \multirow[b]{2}{*}{$\mathrm{N}$} & \multicolumn{4}{|c|}{ Medias estadísticas de dominancia cerebral } \\
\hline & & & $\begin{array}{l}\text { (A) Lógico } \\
\text { Medias }\end{array}$ & $\begin{array}{l}\text { (B) Controlador } \\
\text { Medias }\end{array}$ & $\begin{array}{c}\text { (C) Interpersonal } \\
\text { Medias }\end{array}$ & $\begin{array}{l}\text { (D) Holístico } \\
\text { Medias }\end{array}$ \\
\hline \multirow{5}{*}{ Administrativa } & Planeación & 15 & 28.8 & 25.2 & 21.6 & 24.4 \\
\hline & Finanzas & 4 & 32.2 & 28.2 & 20.5 & 19.0 \\
\hline & Materiales & 10 & 31.9 & 24.8 & 20.7 & 22.6 \\
\hline & \multicolumn{2}{|c|}{ Media Área } & 30.3 & 25.5 & 21.1 & 23.0 \\
\hline & \multicolumn{2}{|c|}{ Desv. Estándar } & 5.0 & 4.2 & 3.7 & 4.7 \\
\hline \multirow{6}{*}{ Manejo de Personal } & Compras & 11 & 27.3 & 26.5 & 22.0 & 24.0 \\
\hline & Rec. Hum. & 13 & 27.0 & 23.8 & 23.2 & 25.9 \\
\hline & Producción & 29 & 30.5 & 26.1 & 19.5 & 23.8 \\
\hline & Seguros & 7 & 26.8 & 29.0 & 22.2 & 21.8 \\
\hline & \multicolumn{2}{|c|}{ Media Área } & 28.7 & 26.0 & 21.1 & 24.1 \\
\hline & \multicolumn{2}{|c|}{ Desv. Estándar } & 6.0 & 4.5 & 4.7 & 4.8 \\
\hline \multirow{6}{*}{ Diseño } & Ingeniería & 46 & 31.6 & 25.4 & 19.5 & 23.3 \\
\hline & Sistemas & 29 & 31.0 & 24.4 & 19.4 & 25.1 \\
\hline & Operaciones & 12 & 30.7 & 26.5 & 18.7 & 24.0 \\
\hline & \multicolumn{2}{|c|}{ Media Área } & 31.3 & 25.3 & 19.4 & 24.0 \\
\hline & \multicolumn{2}{|c|}{ Desv. Estándar } & 4.7 & 4.3 & 4.4 & 4.3 \\
\hline & Total & 176 & $x=30.3, D E=5.3$ & $x=25.6, D E=4.4$ & $x=20.3, D E=4.5$ & $\begin{array}{l}x=23.9 \\
D E=4.5\end{array}$ \\
\hline
\end{tabular}

Tabla 1. Dominancia Cerebral por área laboral de los participantes.

\begin{tabular}{|c|c|c|c|c|c|c|c|c|c|c|}
\hline \multirow{2}{*}{ Factor } & \multirow{2}{*}{ Área } & \multirow{2}{*}{$N$} & \multirow{2}{*}{ Media } & \multirow{2}{*}{$\begin{array}{l}\text { Desviación } \\
\text { estándar }\end{array}$} & \multirow{2}{*}{$\begin{array}{c}\text { Error } \\
\text { estándar }\end{array}$} & \multicolumn{2}{|c|}{ 95\% Interv. de confianza } & \multirow{2}{*}{ Mínimo } & \multirow{2}{*}{ Máximo } & \multirow{2}{*}{$\begin{array}{c}\text { Anova } \\
\text { Unidireccional }\end{array}$} \\
\hline & & & & & & Límite inferior & Límite superior & & & \\
\hline \multirow{4}{*}{ A } & $A D$ & 29 & 30.34 & 5.023 & .933 & 28.43 & 32.26 & 19 & 38 & \multirow{4}{*}{$\begin{array}{c}* F=4.135 \\
\text { Sig. }=0.018\end{array}$} \\
\hline & MP & 60 & 28.75 & 6.075 & .784 & 27.18 & 30.32 & 10 & 40 & \\
\hline & DS & 87 & $31.30^{*}$ & 4.757 & .510 & 30.28 & 32.31 & 16 & 40 & \\
\hline & Total & 176 & 30.27 & 5.378 & .405 & 29.47 & 31.07 & 10 & 40 & \\
\hline \multirow{4}{*}{ B } & $A D$ & 29 & 25.48 & 4.239 & .787 & 23.87 & 27.10 & 19 & 35 & \multirow{4}{*}{$\begin{array}{c}F=0.558 \\
\text { Sig. }=0.573\end{array}$} \\
\hline & MP & 60 & 26.03 & 4.468 & .577 & 24.88 & 27.19 & 16 & 40 & \\
\hline & DS & 87 & 25.26 & 4.325 & .464 & 24.34 & 26.19 & 17 & 38 & \\
\hline & Total & 176 & 25.56 & 4.350 & .328 & 24.92 & 26.21 & 16 & 40 & \\
\hline \multirow{4}{*}{$C$} & $A D$ & 29 & $21.14^{*}$ & 3.729 & .693 & 19.72 & 22.56 & 14 & 29 & \multirow{4}{*}{$\begin{array}{c}* F=3.247 \\
\text { Sig. }=0.041\end{array}$} \\
\hline & $\mathrm{MP}$ & 60 & 21.10 & 4.675 & .604 & 19.89 & 22.31 & 14 & 34 & \\
\hline & DS & 87 & 19.41 & 4.450 & .477 & 18.47 & 20.36 & 12 & 32 & \\
\hline & Total & 176 & 20.27 & 4.478 & .338 & 19.61 & 20.94 & 12 & 34 & \\
\hline \multirow{4}{*}{$D$} & $A D$ & 29 & 23.03 & 4.664 & .866 & 21.26 & 24.81 & 17 & 32 & \multirow{4}{*}{$\begin{array}{c}F=0.626 \\
\text { Sig. }=0.536\end{array}$} \\
\hline & $\mathrm{MP}$ & 60 & 24.12 & 4.798 & .619 & 22.88 & 25.36 & 14 & 38 & \\
\hline & DS & 87 & 24.02 & 4.316 & .463 & 23.10 & 24.94 & 13 & 35 & \\
\hline & Total & 176 & 23.89 & 4.532 & .342 & 23.22 & 24.57 & 13 & 38 & \\
\hline
\end{tabular}

Tabla 2. Análisis de Varianza ANOVA Unidireccional por Dominancia Cerebral ANOVA. 
Los resultados se interpretan como dominante (de 27 a 40 puntos), utilizable (de 15 a 26 puntos) y bajo (de 10 a 14 puntos).

\section{Procedimiento}

Se aplicó el instrumento HBDI a la totalidad del personal gerencial y administrativo. Se analizaron las dominancias cerebrales, según actividades laborales de diez departamentos agrupados en tres áreas laborales, mediante el análisis factorial confirmatorio: (AD, MP, DS). Dicho análisis se reafirmó mediante reuniones gerenciales informativas durante el proyecto. Luego, se describieron los perfiles de dominancia cerebral por área laboral después de obtener la confiabilidad del instrumento y sus propiedades psicométricas. Se analizaron diferencias significativas y se enunciaron, en reunión informativa, las fortalezas y debilidades por cada área de trabajo. En la última reunión, el departamento de Recursos Humanos de la empresa reconoció al instrumento como útil para la selección, promoción, reubicación, detección de necesidades de capacitación y desarrollo organizacional, considerando el clima laboral.

\section{Resultados}

No se encontraron diferencias significativas entre los departamentos agrupados por áreas laborales en cada uno de los cuatro factores de dominancia cerebral, es decir, el comportamiento general de las medias estándar confirmaron la agrupación de las actividades laborales en las tres áreas: AD, MP y DS. En general, los gerentes y administrativos obtuvieron puntuaciones de dominancia en el factor $A(x=30.3 D E=5.3)$, y como utilizables los demás factores, sobresaliendo el factor $B$ $(x=25.6, D E=4.4)$. En puntuaciones mínimas, también en categoría utilizables, se encontró el factor $D(x=23.9, D E=4.5)$, seguido por el $C(x=20.3, D E=4.5)$. Esto indica que el hemisferio cortical superior ( $A-B)$ es mayormente identificado, a partir de actividades laborales documentales-administrativas, con estilos de pensamiento y habilidades lógicas, matemáticas, analíticas y técnicas para la totalidad de los participantes, con debilidades en actividades de trabajo en equipo, relaciones interpersonales y visión holística-creativa, correspondientes al hemisferio límbico inferior (C-D) (tabla 1 Dominancia Cerebral por área laboral de los participantes).

\section{Análisis Diferencial ANOVA-Unidireccional}

Se encontró que el área DS obtuvo mayor dominancia $A(x=$ $31.30)$, seguida por el área de $A D(x=30.34)$ y $M P(x=28.75)$, con diferencia significativa $(F=4.135$, sig. $=0.018)$. El área de diseño utiliza una dominancia cortical superior izquierda, considerada en categoría lógica. Asimismo, para el factor C hubo diferencia al ubicar en puntuaciones mayores consideradas en categoría de dominancia cerebral utilizable el área de $A D(x=21.14)$, luego de $M P(x=21.1)$ y $D S(x=19.4)$ con $\mathrm{F}=3.247$ (sig. $=0.041$ ). El área administrativa activa la dominancia cortical superior derecha en la categoría de controladora. Los factores B y D no resultaron en diferencia significativa por área laboral (figura 1 Dominancia Cerebral por Área Laboral y tabla 2 Análisis de Varianza ANOVA Unidireccional por Dominancia Cerebral).

\section{Análisis Factorial Confirmatorio y Consistencia Interna}

Mediante rotación Varimax Kaiser se obtuvieron 15 factores que agruparon a los 40 indicadores (ítems-reactivos) con una varianza explicada de $81 \%$. Para confirmar la clasificación de Herrmann (1989), se corrieron las rotaciones con cuatro factores correspondientes a cada dominancia cerebral establecida. El resultado de la varianza explicada disminuyó a $75 \%$, sin embargo, se consideró aceptable. Asimismo, se obtuvieron correlaciones significativas positivas entre los ítemsreactivos de cada factor $(p<0.05)$, confirmando la agrupación estadística de los mismos. Por otro lado, se observó correlación negativa significativa producto-momento Pearson entre los factores A-B ( $r=-0.230), A-C(r=-0.673), A-D(r=-.302)$ y B-D ( $r=-$ $.580)$, lo cual indica que cada factor discrimina adecuadamente a partir de los demás. La escala general del Instrumento de Dominancia Cerebral HBDI, obtuvo un alpha de Cronbach de $\alpha=$ 0.657 , en el cual para el factor A la consistencia interna fue relevante $(a=0.075)$, adecuada para $B(\alpha=0.630)$ y en los factores $C(\alpha=0.562)$ y $D(\alpha=0.537)$ la consistencia fue menor, pero aceptable (tabla 3 Análisis Factorial Confirmatorio HDMI).

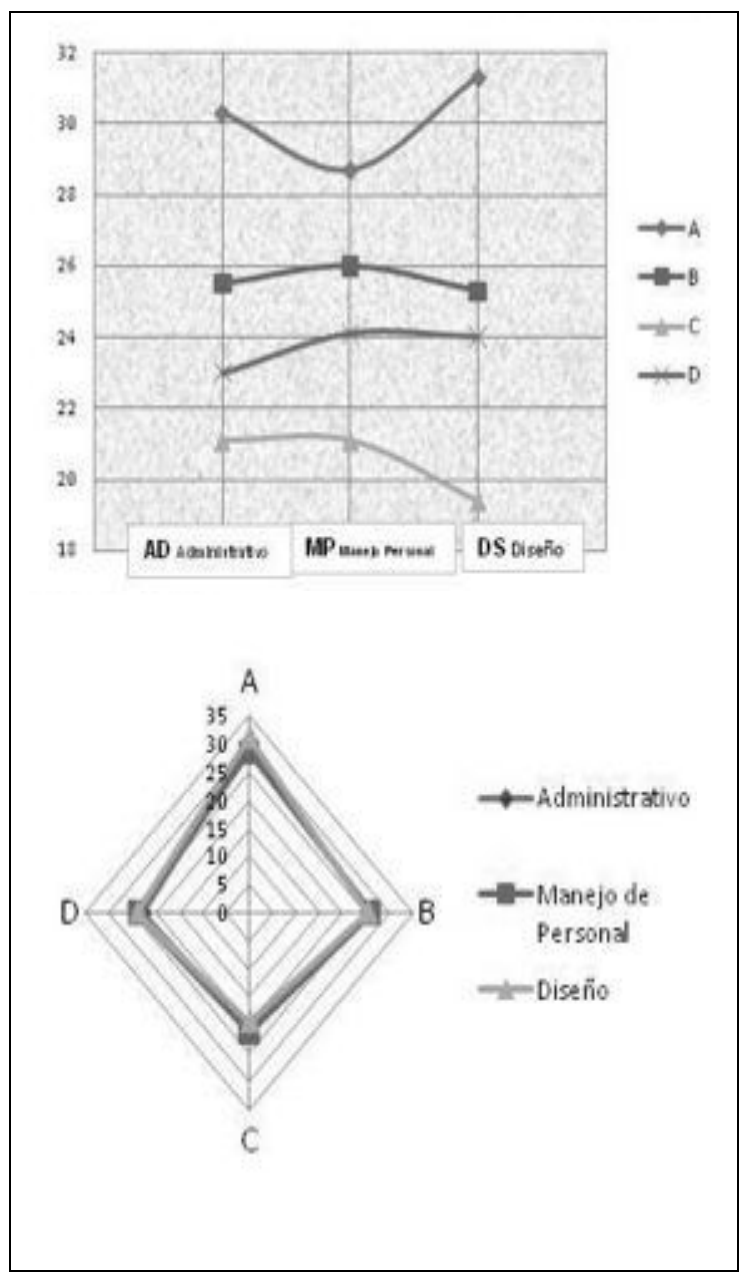


Figura 1. Dominancia Cerebral por Área Laboral Cerebral.

\section{Discusión}

El instrumento Herrmann de Dominancia Cerebral HBDI (1989) mantuvo elementos de confiabilidad para ser utilizado en la evaluación de estilos de pensamiento, preferencias, habilidades y actitudes en los gerentes y administrativos respecto de su área laboral, principalmente en los procesos de selección, promoción y desarrollo organizacional para mejorar la productividad y el ambiente laboral. Al conocer las propiedades psicométricas de factorialidad y fiabilidad del HBDI en una empresa maquiladora en Ciudad Juárez, México, no solo se consideran nuevas posibilidades de evaluación y adecuación persona-puesto (Carter, 1998), sino que se aportan elementos de comprensión en la cultura mexicana laboral local. Algunos investigadores han insistido en la presencia de la cultura instrumental pragmática en la industria manufacturera y una relativa ausencia de humanización o despersonalización en los procesos productivos (Rodríguez, 2000). Así, de acuerdo con Lorens (2005), al conocer aportaciones innovadoras científicas a partir de las neurociencias, es posible construir indicadores cada vez más certeros respecto de estilos de pensamiento y habilidades personales adecuados a los perfiles de puestos empresariales acordes con la localidad, más allá de la aplicación de instrumentos descontextualizados universales.

Los resultados indicaron que los estilos pragmáticos, técnicos, lógicos, matemáticos y controladores son reportados por los gerentes y administrativos de la industria maquiladora juarense como relevantes para la productividad. Así, las puntuaciones altas en los cuadrantes A-lógico y B-controlador, en todas las áreas laborales y departamentos, constituyeron un perfil de dominancia cerebral cortical superior. Esto significa que la productividad está asociada con factores de racionalidad y autogestión de recursos personales para responder a las exigencias laborales de una forma individualista y centrada en resultados debidamente documentados, lo cual se identifica como actividad exitosa y eficiente. Por otro lado, aunque no son puntuaciones débiles, en categoría de utilizables se encontraron los cuadrantes C-interpersonal y D-holístico, en menores puntuaciones respecto de los anteriores. Es decir, las debilidades en el personal gerencial y administrativo mexicanos fueron detectadas con relación al trabajo en equipo, relaciones interpersonales, manejo emocional y uso de procesos creativos en sus actividades laborales. Así, se identificó la necesidad de fortalecer el clima laboral como elemento relevante que permita mayor comodidad, armonía y convivencia laboral de manera colaborativa en procesos principalmente de cambio, de acuerdo con Franklin (2007).

Los resultados de confiabilidad del instrumento con puntuaciones alpha mayores al indicador: $\alpha=0.550$ se corroboraron en reuniones con el departamento de recursos humanos. En ellas se especificó que, aunque mantienen procesos de motivación mediante bonos económicos y realización de actividades sociales familiares, reconocen que la colaboratividad en actividades laborales ha sido difícil de promover o capacitar, principalmente cuando se ejercen orientaciones conductuales de recompensa a la productividad como tal, sin considerar procesos motivacionales del clima laboral. Así, las habilidades de resolución de problemas con exactitud y seguimiento instruccional son ejecutadas por los empleados en forma más individual que colaborativa, principalmente en el área de diseño DS, en la que se esperaría que los ingenieros fuesen más creativos e innovadores, de acuerdo con Penfield y Rasmusen (1957); habilidades resultantes como utilizables y no dominantes, lo cual fue indicado por la diferencia significativa con el análisis de varianza unidireccional.

El departamento de recursos humanos reconoció la eficacia del instrumento de dominancia cerebral. Sin embargo, es necesario considerar un seguimiento puntual mediante réplica para encontrar mayor validación. En este sentido, el presente informe se considera inicial por el impacto que reportó esta industria maquiladora, quedando abierta la posibilidad de realizar estudios comparativos con otras plantas localizadas en el estado de Chihuahua, México, incluso con otras empresas internacionales. Esto permitiría visualizar su uso, no solo en procesos de selección, promoción y adecuación laboral, sino en la comprensión de factores socioculturales motivacionales que constituyen los climas laborales y organizacionales a favor de la convivencia y mejoramiento del estilo de vida, en las dimensiones social, emocional y de salud en general.

\section{Referencias}

Bunderson, C.V., Olsen, J.B., y Herrmann, W.E. (1982). A fourfold model of multiple brain dominance and its validation through correlational research. Scientific and Technical Report \#10: prepared for General Electric. Orem, Utah: Wicat Incorporated Learning Design Laboratories.

Bunderson, C.V., y Olsen, J.B. (1980). A factor analysis of personal profile measures related to cerebral hemisphere specialization. Scientific and Technical Report \#4: prepared for General Electric,. Orem, I tab: WICA T Incorporated Learning Design Laboratories.

Carter, R. (1998). El Nuevo Mapa del Cerebro. Barcelona: Ediciones de la Librería.

Franklin Benjamín, E. (2007). Auditoria Administrativa: gestión estratégica del cambio. 2da ed. México: Pearson Educación, pp. 843.

Gardner, H. (1993). Inteligencias Múltiples. La teoría en la Práctica. Barcelona: Paidós.

Herrmann, Ned. (1989). The creative brain. Bufalo: Brain books.

Herrmann, Ned. (1996). The whole brain business. New York: McGrawHill.

Lorens Fábregas, J. (2005). Gerencia de Proyectos de Tecnología de Información. Caracas, Venezuela: El Nacional, pp. 284.

MacLean, P. (1990) The Triune Brain Evolution. New York: Plenum Press.

Montoya Palacio, A. (2002). Conceptos Modernos de la Administración de Compras. Bogotá, Colombia: Norma, pp. 420.

Morrisey, Georgel. (1996). Planeación Táctica. México: Prentice Hall Hispanoamericana, S.A. de C.V., pp. 133.

Penfield, W. y Rasmusen, T. (1957). The cerebral cortex of men. A clinical Study of Localization of Functions. N.Y.: Mc Millan.

Ramírez Barrera, Mario. (1986). Manual de Técnicas de Supervisión en la Producción. México DF: Limusa, S.A. de C.V., pp.177.

Robbins, P. (1999). Comportamiento Organizacional. México: PrenticeHall.

Rodríguez Ebrad, Luz Angélica. (2000). Administración de Recursos Humanos. Ciudad Juarez Chih, México: ANPEGA, pp. 95.

Sperry, R. (1973). Lateral specialization of cerebral function in the surgically separated hemispheres. New York: Academic Press.

Springer, S.P. y Deutsch, G. (2006). Cerebro izquierdo, cerebro derecho. Barcelona, España: Editorial Gedisa. 